\title{
2D vs 3D morphological analysis of dorsal root ganglia in health and painful neuropathy
}

\author{
Valentina Alda Carozzi, ${ }^{1}$ Chiara Salio, ${ }^{2}$ Virginia Rodriguez Menendez, ${ }^{1}$ Elisa Ciglieri, ${ }^{3}$ Francesco Ferrini ${ }^{2,4}$ \\ ${ }^{1}$ Experimental Neurology Unit, School of Medicine and Surgery, University of Milano-Bicocca, Monza (MB), Italy \\ ${ }^{2}$ Department of Veterinary Sciences, University of Turin, Grugliasco (TO), Italy \\ ${ }^{3}$ Max Planck Institute for Metabolism, Köln, Germany \\ ${ }^{4}$ Department of Psychiatry \& Neuroscience, Université Laval, Québec, QC, Canada
}

Dorsal root ganglia (DRGs) are clusters of sensory neurons that transmit the sensory information from the periphery to the central nervous system, and satellite glial cells (SGCs), their supporting trophic cells. Sensory neurons are pseudounipolar neurons with a heterogeneous neurochemistry reflecting their functional features. DRGs, not protected by the blood brain barrier, are vulnerable to stress and damage of different origin (i.e., toxic, mechanical, metabolic, genetic) that can involve sensory neurons, SGCs or, considering their intimate intercommunication, both cell populations. DRG damage, primary or secondary to nerve damage, produces a sensory peripheral neuropathy, characterized by neurophysiological abnormalities, numbness, paraesthesia and dysesthesia, tingling and burning sensations and neuropathic pain. DRG stress can be morphologically detected by light and electron microscope analysis with alterations in cell size (swelling/atrophy) and in different subcellular compartments (i.e., mitochondria, endoplasmic reticulum, and nucleus) of neurons and/or SGCs. In addition, neurochemical changes can be used to portray abnormalities of neurons and SGC. Conventional immunostaining, i.e., immunohistochemical detection of specific molecules in tissue slices, can be employed to detect, localize and quantify particular markers of damage in neurons (i.e., nuclear expression of ATF3) or SGCs (i.e., increased expression of GFAP), markers of apoptosis (i.e., caspases), markers of mitochondrial suffering and oxidative stress (i.e., 8-OHdG), markers of tissue inflammation (i.e., CD68 for macrophage infiltration) etc. However classical (2D) methods of immunostaining disrupt the overall organization of the DRG, thus resulting in the loss of some crucial information. Whole-mount (3D) methods have been recently developed to investigate DRG morphology and neurochemistry without tissue slicing, giving the opportunity to study the intimate relationship between SGCs and sensory neurons in health and disease. Here, we aim to compare classical (2D) vs whole-mount (3D) approaches to highlight "pros" and "cons" of the two methodologies when analysing neuropathy-induced alterations in DRGs.

Key words: Dorsal root ganglia; sensory neurons; satellite glial cells; painful peripheral neuropathy; whole mount immunolocalization.

Correspondence: Valentina Alda Carozzi, Experimental Neurology Unit, School of Medicine and Surgery, University of Milano-Bicocca, Via Cadore 48, 20900 Monza (MB), Italy. E-mail: valentina.carozzi1@unimib.it

Supplementary Video 1. Representative 3D reconstruction of a whole-mount mouse DRG in which CGRP+ neurons (blue) and SGCs (GS+, red) are immunolabelled.

Supplementary Video 2. Representative 3D reconstruction of a whole-mount mouse DRG showing CGRP+ (blue) and IB4+ (yellow) sensory neurons. 


\section{Introduction}

Dorsal root ganglia (DRGs) are clusters of sensory neurons and satellite glial cells (SGCs) in intimate relationships. They are particularly vulnerable to stress of different origin and intensity due to the lack of the blood brain barrier in the peripheral nervous system. Peripheral neuropathy is the most common disorder of the peripheral nervous system in adults with increasing prevalence and heterogeneous causes. Since no effective treatment is available for patients suffering from this debilitating condition, the identification of molecular pathways is mandatory to elucidate mechanisms of disease and identify novel targets for neuroprotective therapies. Despite the classical immunostaining is a valuable analysis to go insight the morphostructural features as well as neurochemistry of DRG damage, new innovative whole-mount analysis can be valid alternative approaches for studying the 3D morpho-functional abnormalities in sensory neurons, SGCs and in their intimate exchanges. In the following paragraphs, we will: i) recall the distinctive anatomical features of DRGs; ii) revise the common approaches for investigating DRG alterations in peripheral neuropathies; and iii) introduce recently developed whole-mount methodologies. Advantages and disadvantages of the different techniques will be discussed.

\section{Anatomy of the dorsal root ganglia}

\section{Sensory neurons}

Primary sensory neurons of the DRGs are the first-order neurons along the pathway conveying sensory information from the periphery to the central nervous system. They are pseudounipolar neurons with a single axon that bifurcates in a branch projecting to the periphery and a branch projecting to the spinal cord. Sensory neurons detect and transduce information from a variety of specialized receptors, such as nociceptors, mechanoreceptors, and proprioceptors. ${ }^{1}$

Several classifications of sensory neurons have been proposed over the years based on: the cell body size, the neurochemical phenotype, the functional physiological properties, the degree of myelination of peripheral branches and the pattern of central termination in the spinal dorsal horn..$^{2-4}$

Based on the cell body diameter, sensory neurons can be subdivided in small-, medium- and large-sized neurons.

Small-sized DRG neurons (10-30 $\mu \mathrm{m}$ in diameter) give rise to small diameter unmyelinated $\mathrm{C}$ fibers that display a slow conduction velocity and mostly convey noxious and/or thermal stimuli to the CNS. ${ }^{5}$ Based on the phenotype, small neurons can be further split into peptidergic or non-peptidergic neurons depending on the expression of transmitter neuropeptides. ${ }^{3}$ Peptidergic nociceptors express one or more neuropeptides, such as the calcitonin generelated peptide (CGRP), ${ }^{6-10}$ substance P (SP), ${ }^{9-11}$ somatostatin (SST). ${ }^{9} 12$ Further subdivisions of peptidergic neurons have been proposed based on the neurotrophic content (i.e., BDNF and GDNF). ${ }^{13}$ Non-peptidergic nociceptors typically bind the isolectin B4 from Griffonia simplicifolia (IB4) and are immunoreactive to the LA4 antibody. ${ }^{14}$ These neurons also express GDNF receptors RET, GFR $\alpha 1$, GFR $\alpha 2^{9,15,16}$ and the purinergic receptor P2X $3 .{ }^{17}$

Medium-sized DRG neurons (30-50 $\mu \mathrm{m}$ in diameter) are mainly nociceptors and give rise to fine myelinated $\mathrm{A} \delta$-type primary afferent fibers displaying faster conduction velocity as compared to $\mathrm{C}$ fibers. ${ }^{1}$ These neurons may express the neuropeptide CGRP, ${ }^{6,18}$ but do not contain binding sites for IB4. Large-sized DRG neurons ( $>50 \mu \mathrm{m}$ in diameter) give rise to heavily myelinated
A $\beta$-type primary afferent fibers displaying fast conduction velocities. Large neurons typically encode touch or proprioception and express the neurofilament NF200. ${ }^{19}$

The continuous discovery of novel neurochemical and functional markers, ${ }^{4}$ as well as the application of new molecular techniques and single-cell proteomics to DRG neurons ${ }^{20,21}$ have identified additional neuronal phenotypes, including C-fiber small neurons that do not express peptides and do not bind IB4, but express the vesicular glutamate transporter type 3 (VGLUT3), the tyrosine hydroxylase (TH), ${ }^{22-24}$ the chemokine-like protein TAFA4 ${ }^{25,26}$ and the Gai-interacting protein GINIP. ${ }^{23,27,28}$

Functionally, the different neuronal phenotypes have been associated with specific sensory modalities. Indeed, the thermal receptor TRPV1 is mostly found in peptidergic neurons, ${ }^{29}$ while mechanotransducers such as PIEZO1 ${ }^{30}$ and TACAN ${ }^{31}$ are chiefly found in non-peptidergic neurons. On the other hand, recent evidence obtained by in vivo calcium imaging have demonstrated that most small sensory neurons are polymodal. ${ }^{32}$ In addition, important species differences could be observed in phenotypic marker expression. For instance, the vanilloid receptor TRPV1 is expressed in both peptidergic and non-peptidergic sensory neurons in rat, ${ }^{33-37}$ and a small population of non-peptidergic neurons expressing TRPV1 has also been reported in mice. ${ }^{23,37-39}$ In a similar manner, GINIP is exclusively expressed in non-peptidergic neurons in mouse, ${ }^{23,39,40}$ while it has been also described in peptidergic neurons in rat. ${ }^{28}$ Even in human DRGs, the neurochemical boundaries between peptidergic and non-peptidergic populations of sensory neurons are not as sharp as originally described in rodents. ${ }^{41}$ Thus, neuronal phenotypes may be important, but not sufficient, for defining the role of DRG neuronal populations in sensory encoding. Indeed, several lines of evidence strongly indicate that a crucial role in the definition of specific sensory modalities is played by the central arrangement of primary afferent projections in the spinal dorsal horn. ${ }^{42,43}$

\section{Satellite glial cells}

Satellite glial cells (SGCs) of the DRGs are a group of perineuronal cells that envelop cell bodies of sensory neurons providing metabolic support and influencing neuronal function. ${ }^{44-47}$ SGCs were described as cells with a relatively large nucleus and perineuronal processes. ${ }^{46}$ Then, subsequent investigations led to identify SGCs as laminar cells with a flattened glial sheath surrounding sensory neurons. ${ }^{44}$ Specifically, several SGCs surround a single neuron forming a morphological and functional unit, ${ }^{44,48}$ which is separated from adjacent SGCs/neurons units by connective tissue. ${ }^{44,46,47}$ The number of SGCs rises with the increase of the neuron's volume..$^{46,49}$ In some species such as lizard, rat and rabbit, it has been described that a common glia sheet could wrap different neurons forming a "cluster", mostly in young animals. ${ }^{47,50}$ In these units, glial and neuronal membranes are in close apposition, with a distance of 15-20 nm. ${ }^{46}$ The glial layer could also be discontinuous leading to a direct contact between membranes of two adjacent neurons. ${ }^{47,51}$

SGCs around DRG neurons are connected each other by gap junctions consisting of protein connexins ${ }^{46}$ and the most abundant so far described in SGCs are Cx43 and Cx32..$^{52,53}$ Moreover, SGCs show dye coupling as observed after injection with Lucifer yellow. ${ }^{54}$ These features clearly indicate that SGCs carry out several important functions for DRG homeostasis, providing trophic and mechanical support for sensory neurons, regulating ion concentration, diffusion and trafficking of molecules between neurons. $^{48}$

SGCs can be identified by the expression of several glial markers such as glutamine synthetase, ${ }^{51,55}$ GFAP, ${ }^{44,56,57}$ S-100, ${ }^{58,59}$ vimentin, ${ }^{58} \mathrm{Cx} 42,{ }^{53} \mathrm{Kir} 4.1$ potassium channels ${ }^{60}$ and glutamate 
transporters. ${ }^{45,61}$ In particular, Nascimento and colleagues ${ }^{58}$ have postulated the existence of a heterogeneous population of SGCs in DRGs after the observation that the expression pattern of S-100 and vimentin in SGCs is related to the neuron size they enwrapped. The heterogeneity of SGCs is consistent with our recent observation that different neuron-glia arrangements can be found in DRG for specific neuronal phenotypes. ${ }^{51}$

Due to the peculiar neuron-glia arrangement, an increasing number of studies have investigated the potential impact of transglial communication in shaping sensory transmission. The seminal work of Devor and Wall ${ }^{62}$ demonstrated electrical coupling between DRG neurons arranged in small clusters. Subsequently, Rozansky and colleagues ${ }^{63,64}$ showed that the spread of excitation from stimulated sensory neurons to adjacent neurons is mediated by the interposed SGCs. The authors defined this type of neuronglia-neuron communication as "sandwich synapse". Following a train of stimulation, sensory neurons signal to the associated SGCs via purinergic signaling (i.e., through $\mathrm{P} 2 \mathrm{Y} 2$ receptors) ${ }^{64}$ In turn, activated SGCs depolarize adjacent neurons via glutamate release and NMDA receptor activation ${ }^{64}$ or nitric oxide. ${ }^{65}$ The electrical coupling between SGCs via gap junctions may additionally amplify the signal allowing the recruitment of several adjacent neurons.

\section{Conventional approaches for morpho-functional analysis of DRGs during peripheral neuropathy}

The damage of DRG determines the establishment of a sensory neuropathy, which can be due to a primary damage of sensory neurons and/or SGCs (for example after platinum-derived chemotherapy) or to a damage secondary to peripheral nerve injury (for example after peripheral nerve trauma, as compression or transection). Patients with sensory neuropathy may have neurophysiological abnormalities in peripheral nerves, numbness, paraesthesia and dysesthesia, proprioceptive ataxia, tingling and burning sensations, widespread areflexia, neuropathic pain. Several are the causes of peripheral neuropathy, as autoimmune disease (e.g., GuillainBarre syndrome), diabetes, infections, inherited disorders (e.g., Charcot Marie Tooth), solid and blood tumors, alcoholism, exposure to toxic substances (drugs as chemotherapy, poisons, as heavy metals and industrial chemicals), traumas or pressure of the peripheral nerves and vitamin deficiencies (B-1, B-6 and B-12, E). The features of DRG abnormalities can be determined by the nature of the insult and can involve sensory neurons, SGCs or, considering their intercommunication, both cell populations. Traditional immunohistochemical/histological approaches on DRG sections at both optical and ultrastructural level have been extensively used to address both morphological and molecular changes in pathological conditions. In the following paragraphs, we will review the main applications of these approaches in painful peripheral neuropathy.

\section{Alterations in DRG sensory neurons}

Depending on the cause and exposure extension, different cellular compartments can be damaged in sensory neurons. Electron microscopy observations, for example, revealed mitochondria swelling, dilatation in the cisternae of rough endoplasmic reticulum and Golgi complex, microtubule and neurofilament disarranging after chemical exposure. Moreover, neuronal enlargement, rounding of the cytoplasmic membrane, eccentric displacement of the nucleus and nucleolus, loss of Nissl substance can be evident. ${ }^{66,67}$

Neuronal suffering can lead to neuronal death through different pathways that can be biochemically and/or morphologically determined (autophagy, necrosis, apoptosis). Morphological features of necrotic sensory neurons involve cell swelling and degen- eration of cytoplasmic organelles under the action of cellular lytic enzymes (lipases, proteases, endonucleases) that produce alterations in nuclear and cellular membranes, resulting in cellular disgregation and recruitment of inflammatory cells. Depletion of ATP can influence a progressive decrease of cellular processes and organelles' failure. ${ }^{68}$

By contrast, apoptosis requires energy and protein synthesis to proceed and is regulated by several specific molecules that orchestrate the cascade of apoptotic events (i.e., caspases). Morphological features of apoptotic sensory neurons include cytoplasmic shrink, irreversible fragmentation and condensation of chromatin (piknosis) with intact nucleus. Organelles and plasma membranes are unaltered, thus avoiding an inflammatory response. Neurotoxic platinum drugs as cisplatin and oxaliplatin, employed in chemotherapy regimens against several solid tumors (i.e., testis, lung, ovarian, colon cancers), produce a "neuronopathy" with DRG sensory neurons damage ${ }^{69}$ and triggering of apoptotic pathways (Figure 1).

In diabetic sensory neuropathy, peripheral microvascular injury associated with the cellular consequences of high glucose levels in the blood leads to the formation of advanced glycation end products in neurons and increased production of reactive oxygen species (ROS) that alter neuronal metabolism and function. ${ }^{70-}$ ${ }^{74}$ Morphological and quantitative evaluations on DRG of animals affected by experimental diabetic neuropathy showed somatic atrophy $^{75}$ and a disorganized neuronal structure with cellular shrinkage and nuclear and nucleolar blurring with the formation of vacuolar-like structure in the cytoplasm. A computer-assisted quantitative analysis of Nissl/stained neurons also showed an evi-
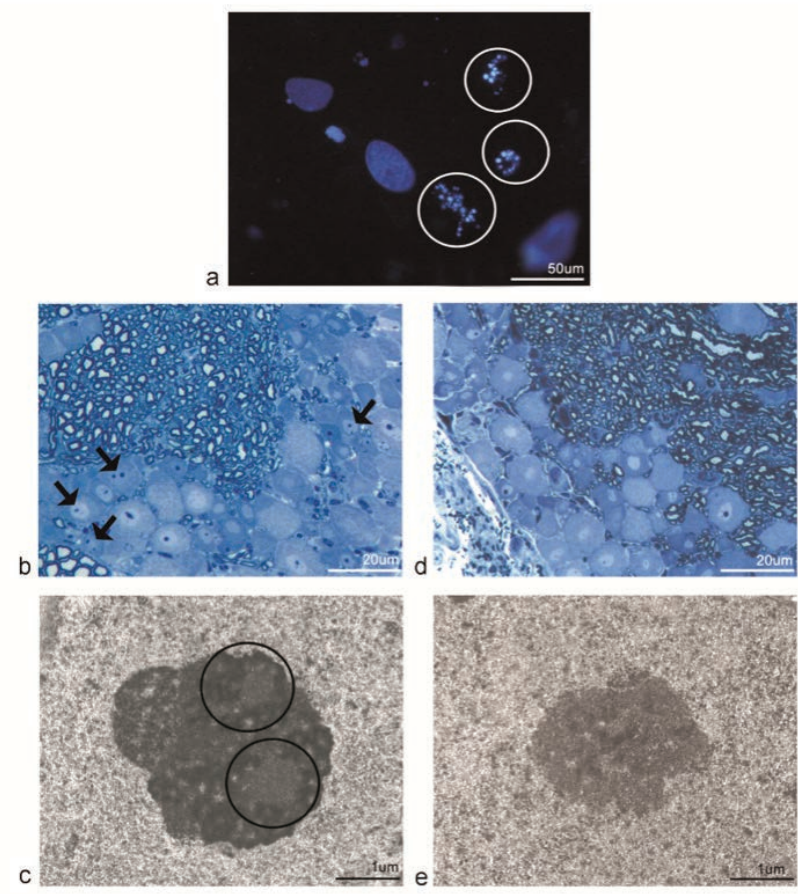

Figure 1. Examples of neuronal alterations detected by standard histological procedures on DRG sections after exposure to neurotoxic chemotherapy (cisplatin): nuclear condensation and fragmentation can be observed in DRG sensory neurons exposed to cisplatin in vitro (a, Hoechst staining). Cisplatin determines an increased incidence of multiple nucleoli in vivo (b) as well as alterations in nucleolar segregation of granular and fibrillar components (c) in DRG sensory neurons compared to non-treated control (d-e). 
dent neuronal loss in the DRG of diabetic neuropathic rats. ${ }^{76}$

The morphofunctional changes of some subpopulations of sensory neurons can be relevant also during neuropathic pain. In the onset of neuropathic pain, peripheral nerve injury increases the excitability of sensory neurons in DRGs leading to spontaneous activation and discharge (hyperexcitability), then involving upper central circuits. Neurochemical properties can be used to identify those neurons who are involved in nociception and are altered due to the establishment of neuropathic pain by immunostaining. For instance, the capsaicin receptor TRPV $1{ }^{77-79}$ can be activated and/or sensitized by mechanical stress, noxious heat, protons, and other endogenous compounds released following tissue injury. TRPV1 activation determines neuronal depolarization, which causes the release of sensory neuropeptides such as CGRP and SP from peripheral and central nerve terminals which in turns enhance the sensitization of nociceptor through the interaction with their effector cell receptors. ${ }^{80}$ As previously stated, the presence of TRPV1 is restricted to small- and medium-sized neurons with reactivity in both peptidergic (CGRP+) and non-peptidergic (CGRP-) sensory neurons. ${ }^{33,81}$ In DRG of neuropathic rats in which neurotoxic chemotherapy determined mechanical and thermal nociception, TRPV1+ neurons were increased and most TRPV1+ neurons had a small-medium size. A similar pattern has been described for CGRP with a decrease in the co-localization with TRPV1. ${ }^{82}$

\section{Alterations in DRG, SGCs and in neuron-SGCs crosstalk}

Although the scenario is huge and conditions variable, SGCs seem to be generally less susceptible than sensory neurons to several kinds of injury. In many cases, the alterations of SGCs do not seem to be due to a direct effect of exogenous insults, rather to the response of these cells to alterations occurring in sensory neurons. Consequently, in most cases, SGCs damage can be described as secondary to sensory neuron damage. The general response of SGCs to toxic-induced neuronal as well as axonal injury is the increase in number (i.e., gliosis) after mitotic division ${ }^{83-85}$ to meet the increased metabolic requirements of damaged neurons to attempt axonal regeneration. After this, SGCs undergo a series of structural modifications involving cytoplasmic organelles and some metabolic changes in enzymatic activities. Evident sign of SGCs activation is an increased expression of GFAP, S100, transforming growth factors, neurotrophins, receptors, cell adhesion molecules, interleukins, tumor necrosis factor. ${ }^{57,59,86-89}$ Other changes that occur in SGCs after neuronal damage are a marked increase in gap junctions that connect neighbouring SGCs and the formation of bridges from previously separated perineuronal sheats. ${ }^{90}$ In physiological conditions, SGCs are connected within the same perineuronal sheats, while in pathological circumstances, the formation of molecular bridges between different perineuronal sheats and the increased gap junction lead to an enhanced coupling among SGCs providing long-distance interconnections between them. These plastic changes in SGCs are trophic for damaged neurons in responding to stress. ${ }^{91}$

Considering that SGCs are the only cells in direct contact with sensory neurons and fundamental in regulating neuronal homeostasis and excitability, they play a role in the development and maintenance of neuropathic pain. Their changes are secondary to nervous alterations, after activation of injured neurons-SGCs signalling pathways. Several molecular mechanisms have been proposed to explain how SGCs can be activated and react to nerve injury and sensory neurons' hyperexcitability acting on the development and maintenance of neuropathic pain. An example is the activation of purinergic receptors on SGCs after ATP release by neurons: this can cause the release of proinflammatory cytokines by SGCs that in turns enhance neuronal excitability. ${ }^{86}$
Since gap junctions between SGCs increase in number and coupling after nerve injury, they can be involved in the generation and maintenance of neuropathic pain. The evidence came from the ability of gap junction blockers in restoring neuronal excitability and reducing pain in several pain models including chemotherapy treatment. ${ }^{92,93}$ Several papers also postulated that the expression of connexins, the proteins that assemble together forming the gap junctions, can be augmented in painful pathological conditions. ${ }^{48,91,94}$ Morpho-structural abnormalities are also evident in SGCs of animals chronically treated with chemotherapy treatment (bortezomib or cisplatin): they show clear cytoplasmic vacuolation and hypertrophy of the cytoplasm, invaginations of the plasmamembrane and altered chromatin segregation, as well as increased proliferation (gliosis). ${ }^{69,95,96}$

GFAP expression increment, because of gliosis, is widely used as a marker of neuronal damage in both CNS and PNS.97-101 For instance, some authors have observed an increment in the number of GFAP+ SGCs surrounding stressed neurons (therefore ATF3 positive) in animal models of arthritis evidencing a direct relationship between neuronal insult and SGCs activation. ${ }^{99,102}$ On the other hand, Barragán-Iglesias and colleagues ${ }^{103}$ and Peters and colleagues ${ }^{104}$ have observed a strong ATF3 positivity also on GFAP+ SGCs at late stages of both diabetic- and paclitaxel-induced peripheral neuropathy animal models.

In addition, Peters and colleagues ${ }^{104}$ reported the presence of CD68+ macrophages in rat DRGs and sciatic nerves after 6 days of paclitaxel treatment that, besides SGCs activation, represents as well a sign of tissue damage. Painful neuropathies induced by paclitaxel activate the expression of monocyte chemotactic protein 1 (MCP-1) triggering macrophage infiltration in the DRG, which cells, in turn, express inflammatory cytokines. ${ }^{105,106}$ Interestingly, this event seems to happen in concomitance with the onset of the neuropathy, suggesting an important role of CD68+ macrophages in the development of the pathology in DRG. This observation is validated, somewhat, by Zhang and colleagues ${ }^{106}$ who describe that the administration of anti-MCP-1 neutralizing antibodies reduced macrophage infiltration in the DRG and prevented the development of mechanical hypersensitivity (paw withdrawal threshold) in the paclitaxel treated animals. Therefore, macrophage infiltration in DRG during painful neuropathy, more than simply helping remove degenerating neuronal debris and contribute to subsequent regeneration, as previously suggested, ${ }^{106,107}$ seems to contribute to the onset of the pathology and maintenance of chronic pain. ${ }^{106,108}$

DRG insult can also trigger the expression of pro- and antiapoptotic molecules. In fact, in a 10-month rat chronic model of diabetes, even though Kamiya and colleagues ${ }^{109}$ observed an increment in pro-apoptotic protein expression (caspase-3) against a decrement (HSP70) or no alteration (Bcl-xL, HSP27) in the antiapoptotic ones, they did not discern the presence of apoptotic cells (DRG neurons, mitochondria or endoplasmic reticulum did not show any morphological alteration). DRG neurons seemed to be kept in a sustained stressed state. Only the Golgi apparatus appeared highly vacuolized and fragmented, and this could have been one of the reasons for the progressive DRG cells loss in these animals. ${ }^{109}$ On the other hand, in chronic (12 months) experimental diabetic rats several authors reported apoptosis (Caspase- 3 and TUNEL positive) as soon as 1 and 3 months after streptozotocin treatment together with the presence of swelled mitochondria with disrupted inner cristae structure and condensation of chromatin. ${ }^{74,110}$ It has also been implied the existence of a direct association between oxidative injury (8-OHdG expression) and neuronal apoptosis in these animals. ${ }^{74}$ Mitochondria of DRG neurons also happen to be a target in platinum-based peripheral neuropathies as demonstrated in vitro ${ }^{111}$ and in vivo ${ }^{112}$ and when damaged ROS rise and calcium homeostasis becomes altered. ${ }^{113,114}$ In fact, platinum- 
based drugs form DNA adducts not only in the nucleus of the DRG neurons, ${ }^{96,115,116}$ but also within mitochondrial DNA where profound functional modifications such as replication and transcription interference take place, leading to a progressive mitochondrial vacuolization and degradation. ${ }^{117}$ Moreover, Corsetti and colleagues ${ }^{118}$ described mitochondrial and cytoplasmic alterations also in SGCs after cisplatin treatment and propose that neuronal damage is subsequent to SGCs damage, therefore attributing to SGCs a main role on platinum-based peripheral neuropathies.

Another marker for SGCs functional changes under stress conditions is Connexin 43. Its expression in gap junctions increases in SGCs after taxane treatment, ${ }^{93}$ indicating an increment of gap junction-mediated coupling between SGCs that contributes to the establishment of chronic pain as reported in different neuropathic pain models. ${ }^{54,90,119,120}$

Moreover, SCG purinergic P2 receptors are the target of the ATP and TNF- $\alpha$ released by stressed or damaged neurons that triggers a gap junction-dependent $\mathrm{Ca}^{2+}$ waves between the SGCs..$^{94,12}$ Interestingly, in diabetes animal models, the purinergic P2X7 receptor is upregulated in SGCs which results in a hyperexcitability of DRG neurons. ${ }^{122}$

In keeping, peripheral neuropathy induces a variety of alterations in both DRG neurons and glia and in their reciprocal interactions that can be investigated by standard histological procedures on DRG sections, combined with immunohistochemistry. These classical approaches provide an effective tool to address cellular and subcellular alterations and still represent the gold standard to detect neurodegenerative processes. However, these classical methods, while favoring the access to individual cell bodies, disrupt the overall organization of the DRG, thus resulting in loss of some crucial information. Optimizing the technical approach to investigate the entire DRG is the necessary premise to fully evaluate the impact of internal organization on function. In recent times, innovative whole-mount methods, discussed below, have been developed to investigate large areas of the nervous system without slicing and allowing the evaluation of cell populations in their intimate relationship, close to physiological conditions.

\section{Whole mount morphological approaches for studying neuron-to-glia structural association in DRGs}

Most whole-mount 3D methods aim to convert large opaque pieces of tissue in transparent specimens by reducing light scattering (tissue clearing methods, for review ${ }^{123,124}$ ). Different technical approaches have been proposed, based on either the use of solvent (hydrophobic methods, such as BBAB, DISCO), aqueous reagents (hydrophilic methods, such as SeeDB), or hydrogels (such as CLARITY). ${ }^{124}$

Initial attempts to clarify nervous tissues were chiefly based on solvent-based approaches to solvate lipid content in the samples, thus allowing the tissues to become translucent. Although relatively easy to implement, these methods were typically hampered by high levels of autofluorescence, incapacity to preserve endogenous fluorescence and limited compatibility with macromolecule-based phenotyping techniques. ${ }^{125}$ Nevertheless, hydrophobic clearing methods have been successfully applied to certain whole-mount preparations of peripheral sensory neurons. ${ }^{123}$ Indeed, a combination of transparency and immunophenotyping has been recently reported in intact DRGs from both embryonic ${ }^{126}$ and adult mice. ${ }^{127}$

On the other hand, the innovative hydrogel based methods proposed by Karl Deisseroth's lab in 2013, known as CLARITY, paved a novel path for combining whole-mount preparations and immunostaining. ${ }^{128}$ The clearing method originally consisted in an active lipid extraction procedure allowing the conversion of entire pieces of brain into light-permissive macromolecule-permeable specimens. ${ }^{128}$ Hydrogel reinforcement allows excellent transparency of the specimen (as obtained by classical hydrophobic methods) with minimal loss of macromolecules and antigenicity. ${ }^{124}$ Moreover, unlike hydrophobic approaches, CLARITY does not affect endogenous fluorescence and can be conveniently adopted in transgenic animals. The initial method was, however, somehow labored and changes have been subsequently proposed to improve reproducibility, reducing costs, and mitigate the overall duration of the clearing/staining protocols (see for instance, PACT, SHIELD, PARS). ${ }^{129}$

Recently, Bernal and colleagues ${ }^{130}$ have proposed a cost-effective variation of CLARITY (inCLARITY) based on passive lipid clearing protocol to image DRGs. The method consisted in a first phase of hydrogel polymerization in acrylamide/bisacrylamide, followed by a long lipid clearing phase in sodium dodecyl sulphate ( $>7$ days). This method was used for combining immunophenotyping of sensory neurons and retrograde fluorescent tracers. While inexpensive and technically simple, the method requires long incubation times spanning over two weeks for both clearing procedures and antibody incubation.

In 2016, we proposed an alternative whole-mount method for 3D DRG processing without a clearing approach. ${ }^{131}$ Indeed, tissue clearing is instrumental to minimize light scattering in large specimens. However, rodent (and particularly mouse) DRGs are relatively small $(<1 \mathrm{~mm})$ and light permissive. The main obstacle to obtain multilayer immunostaining in entire DRGs is thus represented by antibody penetration. To overcome this problem, we proposed to incubate freshly dissected DRGs with collagenase (5-10 $\mathrm{mg} / \mathrm{ml}$ ) to digest the connective tissue forming the DRG capsule. Collagenase was previously used to permit patch clamp recordings in entire rat DRGs. ${ }^{132,133}$ Indeed, we applied this approach for recording functional properties of sensory neurons without dissociating, thus preserving local anatomical interactions. After recording, and following a short fixation in paraformaldehyde, DRGs were processed for immunohistochemistry in order to phenotype the recorded neurons. Surprisingly, we found that collagenase treatment significantly improved antibody penetration in the DRGs, as compared to untreated DRGs. ${ }^{131}$ Representative 3D reconstructions are shown in Supplementary material.

The protocol was subsequently optimized for 3D morphological analysis and the method allowed to uncover specific spatial arrangements of sensory neurons belonging to different cell populations. ${ }^{51}$ Namely, non-peptidergic neurons were found to form cell clusters in which cell somata lie in close apposition, while peptidergic neurons do not. In the same study, we also analyzed the relationship between SGCs and sensory neurons taking advantage of the possibility to analyze the contact between glia and neurons all along the neuronal surface in whole mount-preparation (Figure 2). Interestingly, whole-mount preparation allows us to appreciate how different neuron-glia arrangements can be detected for different populations of sensory neurons. ${ }^{51}$

As compared to clearing-based whole-mount approaches, our proposed protocol is fast, cost effective and highly reproducible and can be easily combined with functional approaches (i.e., addressing the phenotype of recorded neurons).

Major limitations are common to all whole-mount approaches, and specifically:

1) Technical limitations to image large $3 D$ specimens. The increased interest in whole-mount approaches has been accompanied in recent years by a parallel development of optical systems to see deeper in the sample, such as lightsheet microscopy and multiphoton confocal microscopy. ${ }^{134}$ Although these devices are becoming more and more accessible, yet standard one-photon 
scanning confocal microscopes are by far the more common set-up found in many laboratories. The use of one-photon confocal microscopy allows accurate $3 \mathrm{D}$ reconstruction of small volumes or of restricted areas of large samples. ${ }^{135}$ This approach is suitable for mouse DRGs as their diameter falls below the millimeter and long working distance objectives are adequate to fully scan the specimen. In our study, we successfully acquired 3D images with a standard one-photon laser scanning confocal (Leica SP5). Interestingly, a one-photon microscope was also used to image cleared DRG by inCLARITY. ${ }^{130}$ The overall yield in terms of image definition and thickness of 3D reconstruction are comparable in the two studies thus suggesting that the major limiting factor for Z-scanning in DRGs is not due to the use of clearing approaches but, rather, to the technical limitations of the optical devices.

2) Technical issues for analyzing 3D images. 3D reconstruction of biological samples is providing novel insight in the understanding of the organization of the nervous system. However, to properly analyze and extract quantitative information from these samples traditional 2D methods are limited and appropriate software-based methods are required. To this aim, we have developed a software (3DRG), that provides a fully-automated segmentation of positively labeled nociceptors in whole-mount DRG preparations. ${ }^{136}$ 3DRG has been conceived to characterize the sample in a fully-automated way, allowing the visualization of identified cell populations to highlight spatial relations. Recently, Bennett's group has proposed an image analysis tool (StereoMate) to develop image segmentation protocols in DRGs and extract unbiased information at the cell level. ${ }^{127}$ The tool can be combined with a machine learning classifier to facilitate correct object recognition.

$a_{\text {control }}$
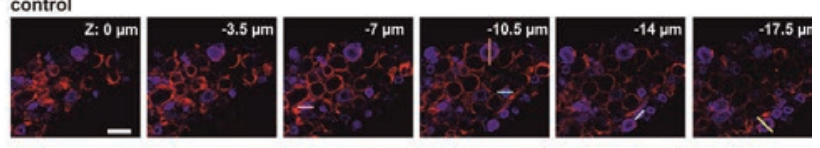

b $_{\text {diabetic }}$
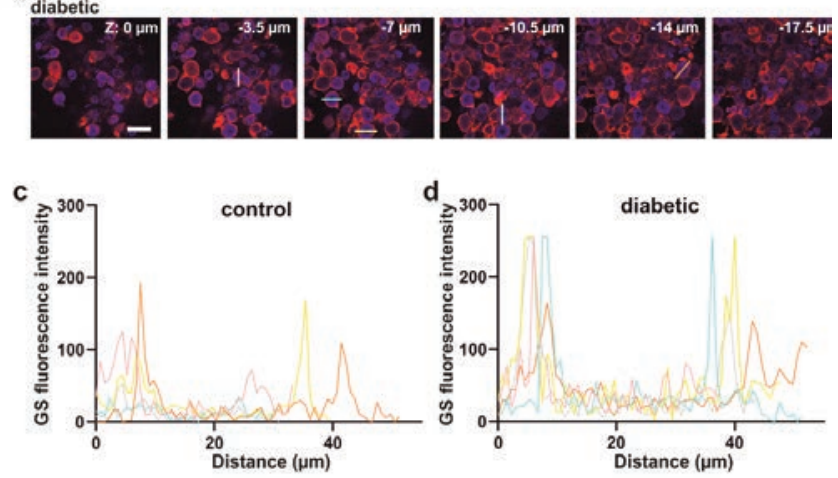

Figure 2. Whole-mount mouse DRG staining after collagenase treatment. a) CGRP+ neurons (in blue) and GS + satellite glial cells (in red) in lumbar DRG from a control mouse; from left to right, sequential z-stacks are shown; a viable approach for GS fluorescence quantitation is also represented; the intensity of GS staining is measured across the maximum diameter along the $\mathrm{Z}$ axis of identified CGRP+ cells (coloured lines).(b) CGRP+ neurons (in blue) and GS+ satellite glial cells (in red) from a diabetic mouse. c-d) GS fluorescence intensity per pixel along the coloured lines (distance in microns) in (a) and (b). Note the fluorescence peaks at the perimeter of the sensory neurons where SGCs are located. Higher fluorescent signals are detected in diabetic mice (d). Scale bar: $50 \mu \mathrm{m}$.
Other machine learning approaches are emerging ${ }^{137}$ and represent a promising avenue for addressing cell-to-cell interactions in a $3 \mathrm{D}$ environment.

\section{Application of a 3D DRG approach in pathologi- cal settings}

While traditional approaches based on classical tissue slicing techniques are still the gold standard for fine subcellular analysis of morphological and molecular alterations in pathological DRGs (Figure 1), yet whole-mount methods represent a powerful and complementary alternative to explore changes in cell-to-cell relationships. Main advantages of 2D vs 3D approaches are reported in Table 1 .

We have recently applied our whole-mount technique to interrogate the impact of diabetic neuropathy in altering neuron-neuron and neuron-glia interactions..$^{51}$ An example of this application and its potentiality is shown in Figure 2. In agreement with previous studies, ${ }^{98}$ major differences were mainly detected in the relationship between SGCs and neurons. Specifically, we used our 3D method to analyze the distribution of SGCs marker GS around identified populations of sensory neurons, such as CGRP neurons (blue in Figure $2 \mathrm{a}, \mathrm{b}$ ). The possibility of changing the $\mathrm{z}$-axis allows to standardize GS quantification around the largest diameter of the identified neurons, which would not be possible in standard cryostat or microtome-based sections, thus making comparisons between groups more robust. Our analysis demonstrated that GS staining is significantly increased around $\mathrm{CGRP}^{+}$neurons from diabetic mice (Figure $2 \mathrm{c}, \mathrm{d}$ ).

$3 \mathrm{D}$ approaches have also been successfully applied to detect DRG alterations following peripheral nerve injury. Indeed, whole mount DRG preparations were successfully used to quantify the loss of neurons in a murine model of spared nerve injury. ${ }^{127} \mathrm{~A}$ similar result can be also obtained by standard stereological approaches, which are, however, time consuming and more exposed to sampling errors. ${ }^{127}$

In perspective, other potential applications of 3D DRG specimens in pathological conditions may include:

- analysis of DRG infiltrations of immune cells;

- structural plasticity of fluorescent-tagged populations of sensory neurons (i.e., change in size or in cell-to-cell relationship);

- alterations in the somatotopic organization of afferent inputs (i.e., by retrograde labelling of sensory neurons innervating restricted peripheral territories);

- identification of functional interaction between neighbouring

Table 1. Pros and cons of whole-mount methods $v$ s. classical section-based approaches.

\begin{tabular}{lll}
\hline PROS AND CONS & 2D methods & 3D methods \\
\hline Costs & + & $++>+++$ \\
Time consuming & ++ & $+>+++$ \\
Neuronal phenotyping & ++ & +++ \\
Neuron-glia relationship & + + & +++ \\
Cell count & ++ & +++ \\
Volumetric analysis & + - & +++ \\
Membrane localizations & +++ & +++ \\
Subcellular localizations & +++ & + \\
Nuclear alterations & +++ & + \\
Coupling with & + - & ++ \\
functional studies & & \\
$\begin{array}{l}\text { Data extraction and } \\
\text { analysis }\end{array}$ & ++ & + \\
\hline
\end{tabular}

++ , highly appropriate; + , appropriate; + , appropriate (but not first choice);,- inadequate. 
sensory neurons by using markers of neuronal activation (i.e., ERK1/2 phosphorylation); ${ }^{138}$

- combining functional and morphological analysis in entire DRGs. ${ }^{131}$

\section{Conclusions}

In keeping, 3D analysis of sensory neurons is a growing and promising method that can be adopted together with or as alternative to standard histological 2D approaches for tackling specific questions around the impact of DRG structure on sensory function (Table 1). The anatomical organization of the DRGs suggests that neuron-neuron as well SGC-SGC or SGC-neuron interactions influence neuron physiology. This "interactive" homeostasis can be lost once the DRG are processed with tissue and/or cell preparation standard protocols for conventional morpho-functional analysis. In the whole 3D DRG, instead, the cyto-architecture of the organ as well as the anatomical relationship between the different cell populations are preserved.

\section{Acknowledgements}

We are thankful to Prof. Arianna Scuteri who kindly provided the picture of cultured neurons tested for nuclear fragmentation. Thanks to Fondazione Cassa di Risparmio di Torino (CRT; grant No. $R F=2015.1690$ to F.F.).

\section{References}

1. Basbaum AI, Bautista DM, Scherrer G, Julius D. Cellular and molecular mechanisms of pain. Cell 2009;139:267-84.

2. Averill S, McMahon SB, Clary DO, Reichardt LF, Priestley JV. Immunocytochemical localization of trkA receptors in chemically identified subgroups of adult rat sensory neurons. Eur J Neurosci 1995;7:1484-94.

3. Lawson SN. Morphological and biochemical cell types of sensory neurons. In: Scott SA, editor. Sensory neurons: Diversity, development, and plasticity. New York: Oxford University Press; 1992. p. 27-59.

4. Le Pichon CE, Chesler AT. The functional and anatomical dissection of somatosensory subpopulations using mouse genetics. Front Neuroanat 2014;8:21.

5. Lawson SN, Perry MJ, Prabhakar E, McCarthy PW. Primary sensory neurones: neurofilament, neuropeptides, and conduction velocity. Brain Res Bull 1993;30:239-43.

6. Gibson SJ, Polak JM, Bloom SR, Sabate IM, Mulderry PM, Ghatei MA, et al. Calcitonin gene-related peptide immunoreactivity in the spinal cord of man and of eight other species. J Neurosci 1984;4:3101-11.

7. Lawson SN. Neuropeptides in morphologically and functionally identified primary afferent neurons in dorsal root ganglia: substance P, CGRP and somatostatin. Prog Brain Res 1995;104:161-73.

8. McCarthy PW, Lawson SN. Cell type and conduction velocity of rat primary sensory neurons with calcitonin gene-related peptide-like immunoreactivity. Neuroscience 1990;34: 623-32.

9. Salio C, Ferrini F, Muthuraju S, Merighi A. Presynaptic modulation of spinal nociceptive transmission by glial cell linederived neurotrophic factor (GDNF). J Neurosci 2014;34: 13819-33.
10. Salio C, Lossi L, Ferrini F, Merighi A. Neuropeptides as synaptic transmitters. Cell Tissue Res 2006;326:583-98.

11. McCarthy PW, Lawson SN. Cell type and conduction velocity of rat primary sensory neurons with substance P-like immunoreactivity. Neuroscience 1989;28:745-53.

12. Ribeiro-da-Silva A. Ultrastructural features of the colocalization of calcitonin gene related peptide with substance $\mathrm{P}$ or somatostatin in the dorsal horn of the spinal cord. Can J Physiol Pharmacol 1995;73:940-4.

13. Salio C, Ferrini F. BDNF and GDNF expression in discrete populations of nociceptors. Ann Anat 2016;207:55-61.

14. Stucky CL, Lewin GR. Isolectin B(4)-positive and -negative nociceptors are functionally distinct. $\mathrm{J}$ Neurosci 1999;19:6497-505.

15. Bennett DL, Michael GJ, Ramachandran N, Munson JB, Averill S, Yan Q, et al. A distinct subgroup of small DRG cells express GDNF receptor components and GDNF is protective for these neurons after nerve injury. J Neurosci 1998; 18:3059-72.

16. Ferrini F, Salio C, Boggio E, Merighi A. Interplay of BDNF and GDNF in the mature spinal somatosensory system and its potential therapeutic relevance. Curr Neuropharmacol 2021;19:1225-45.

17. Bradbury EJ, Burnstock G, McMahon SB. The expression of P2X3 purinoreceptors in sensory neurons: effects of axotomy and glial-derived neurotrophic factor. Mol Cell Neurosci 1998;12:256-68.

18. Lawson SN, McCarthy PW, Prabhakar E. Electrophysiological properties of neurones with CGRP-like immunoreactivity in rat dorsal root ganglia. J Comp Neurol 1996;365:355-66.

19. Lawson SN, Harper AA, Harper EI, Garson JA, Anderton BH. A monoclonal antibody against neurofilament protein specifically labels a subpopulation of rat sensory neurones. J Comp Neurol 1984;228:263-72.

20. Usoskin D, Furlan A, Islam S, Abdo H, Lonnerberg P, Lou D, et al. Unbiased classification of sensory neuron types by large-scale single-cell RNA sequencing. Nat Neurosci 2015;18:145-53.

21. Zheng Y, Liu P, Bai L, Trimmer JS, Bean BP, Ginty DD. Deep sequencing of somatosensory neurons reveals molecular determinants of intrinsic physiological properties. Neuron 2019; 103:598-616.e7.

22. Li L, Rutlin M, Abraira VE, Cassidy C, Kus L, Gong S, et al. The functional organization of cutaneous low-threshold mechanosensory neurons. Cell 2011;147:1615-27.

23. Salio C, Aimar P, Malapert P, Moqrich A, Merighi A. Neurochemical and ultrastructural characterization of unmyelinated non-peptidergic C-nociceptors and C-low threshold mechanoreceptors projecting to lamina II of the mouse spinal cord. Cell Mol Neurobiol 2021;41:247-62.

24. Seal RP, Wang X, Guan Y, Raja SN, Woodbury CJ, Basbaum $\mathrm{AI}$, et al. Injury-induced mechanical hypersensitivity requires C-low threshold mechanoreceptors. Nature 2009;462:651-5.

25. Delfini MC, Mantilleri A, Gaillard S, Hao J, Reynders A, Malapert P, et al. TAFA4, a chemokine-like protein, modulates injury-induced mechanical and chemical pain hypersensitivity in mice. Cell Rep 2013;5:378-88.

26. Kambrun C, Roca-Lapirot O, Salio C, Landry M, Moqrich A, Le Feuvre Y. TAFA4 reverses mechanical allodynia through activation of GABAergic transmission and microglial process retraction. Cell Rep 2018;22:2886-97.

27. Gaillard S, Lo Re L, Mantilleri A, Hepp R, Urien L, Malapert $\mathrm{P}$, et al. GINIP, a Galphai-interacting protein, functions as a key modulator of peripheral GABAB receptor-mediated 
analgesia. Neuron 2014;84:123-36.

28. Liu Z, Wang F, Fischer G, Hogan QH, Yu H. Peripheral nerve injury induces loss of nociceptive neuron-specific Galphaiinteracting protein in neuropathic pain rat. Mol Pain 2016;12:1744806916646380

29. Cavanaugh DJ, Chesler AT, Braz JM, Shah NM, Julius D, Basbaum AI. Restriction of transient receptor potential vanilloid-1 to the peptidergic subset of primary afferent neurons follows its developmental downregulation in nonpeptidergic neurons. J Neurosci 2011;31:10119-27.

30. Wang J, La JH, Hamill OP. PIEZO1 is selectively expressed in small diameter mouse DRG neurons distinct from neurons strongly expressing TRPV1. Front Mol Neurosci 2019;12:178.

31. Beaulieu-Laroche L, Christin M, Donoghue A, Agosti F, Yousefpour N, Petitjean H, et al. TACAN is an ion channel involved in sensing mechanical pain. Cell 2020;180:95667.e17.

32. Wang F, Belanger E, Cote SL, Desrosiers P, Prescott SA, Cote DC, et al. Sensory afferents use different coding strategies for heat and cold. Cell Rep 2018;23:2001-13.

33. Guo A, Vulchanova L, Wang J, Li X, Elde R. Immunocytochemical localization of the vanilloid receptor 1 (VR1): relationship to neuropeptides, the $\mathrm{P} 2 \mathrm{X} 3$ purinoceptor and IB4 binding sites. Eur J Neurosci 1999;11:946-58.

34. Hwang SJ, Oh JM, Valtschanoff JG. Expression of the vanilloid receptor TRPV1 in rat dorsal root ganglion neurons supports different roles of the receptor in visceral and cutaneous afferents. Brain Res 2005;1047:261-6.

35. Michael GJ, Priestley JV. Differential expression of the mRNA for the vanilloid receptor subtype 1 in cells of the adult rat dorsal root and nodose ganglia and its downregulation by axotomy. J Neurosci 1999;19:1844-54.

36. Tominaga M, Caterina MJ, Malmberg AB, Rosen TA, Gilbert $\mathrm{H}$, Skinner K, et al. The cloned capsaicin receptor integrates multiple pain-producing stimuli. Neuron 1998;21:531-43.

37. Woodbury CJ, Zwick M, Wang S, Lawson JJ, Caterina MJ, Koltzenburg M, et al. Nociceptors lacking TRPV1 and TRPV2 have normal heat responses. J Neurosci 2004;24:6410-5.

38. Breese NM, George AC, Pauers LE, Stucky CL. Peripheral inflammation selectively increases TRPV1 function in IB4positive sensory neurons from adult mouse. Pain 2005;115:37-49.

39. Zwick M, Davis BM, Woodbury CJ, Burkett JN, Koerber HR, Simpson JF, et al. Glial cell line-derived neurotrophic factor is a survival factor for isolectin B4-positive, but not vanilloid receptor 1-positive, neurons in the mouse. J Neurosci 2002;22:4057-65.

40. Reynders A, Moqrich A. Analysis of cutaneous MRGPRD free nerve endings and C-LTMRs transcriptomes by RNAsequencing. Genom Data 2015;5:132-5.

41. Shiers SI, Sankaranarayanan I, Jeevakumar V, Cervantes A, Reese JC, Price TJ. Convergence of peptidergic and non-peptidergic protein markers in the human dorsal root ganglion and spinal dorsal horn. J Comp Neurol 2021;529:2771-88.

42. Cavanaugh DJ, Lee H, Lo L, Shields SD, Zylka MJ, Basbaum AI, et al. Distinct subsets of unmyelinated primary sensory fibers mediate behavioral responses to noxious thermal and mechanical stimuli. Proc Natl Acad Sci USA 2009;106:9075-80.

43. Ferrini F, Perez-Sanchez J, Ferland S, Lorenzo LE, Godin AG, Plasencia-Fernandez I, et al. Differential chloride homeostasis in the spinal dorsal horn locally shapes synaptic metaplasticity and modality-specific sensitization. Nat Commun
2020;11:3935

44. Hanani M. Satellite glial cells in sensory ganglia: from form to function. Brain Res Brain Res Rev 2005;48:457-76.

45. Huang LY, Gu Y, Chen Y. Communication between neuronal somata and satellite glial cells in sensory ganglia. Glia 2013;61:1571-81.

46. Pannese E. The satellite cells of the sensory ganglia. Adv Anat Embryol Cell Biol 1981;65:1-111.

47. Pannese E, Ledda M, Arcidiacono G, Rigamonti L. Clusters of nerve cell bodies enclosed within a common connective tissue envelope in the spinal ganglia of the lizard and rat. Cell Tissue Res 1991:264:209-14.

48. Hanani M, Spray DC. Emerging importance of satellite glia in nervous system function and dysfunction. Nat Rev Neurosci 2020;21:485-98.

49. Ledda M, De Palo S, Pannese E. Ratios between number of neuroglial cells and number and volume of nerve cells in the spinal ganglia of two species of reptiles and three species of mammals. Tissue Cell 2004;36:55-62.

50. Pannese E, Procacci P, Ledda M, Conte V. Age-related reduction of the satellite cell sheath around spinal ganglion neurons in the rabbit. J Neurocytol 1996;25:137-46.

51. Ciglieri E, Vacca M, Ferrini F, Atteya MA, Aimar P, Ficarra $\mathrm{E}$, et al. Cytoarchitectural analysis of the neuron-to-glia association in the dorsal root ganglia of normal and diabetic mice. J Anat 2020;237:988-97.

52. Manteniotis S, Lehmann R, Flegel C, Vogel F, Hofreuter A, Schreiner BS, et al. Comprehensive RNA-Seq expression analysis of sensory ganglia with a focus on ion channels and GPCRs in Trigeminal ganglia. PLoS One 2013;8:e79523.

53. Procacci P, Magnaghi V, Pannese E. Perineuronal satellite cells in mouse spinal ganglia express the gap junction protein connexin 43 throughout life with decline in old age. Brain Res Bull 2008;75:562-9.

54. Huang TY, Cherkas PS, Rosenthal DW, Hanani M. Dye coupling among satellite glial cells in mammalian dorsal root ganglia. Brain Res 2005;1036:42-9.

55. Weick M, Cherkas PS, Hartig W, Pannicke T, Uckermann O, Bringmann A, et al. P2 receptors in satellite glial cells in trigeminal ganglia of mice. Neuroscience 2003;120:969-77.

56. Li L, Zhou XF. Pericellular Griffonia simplicifolia I isolectin B4-binding ring structures in the dorsal root ganglia following peripheral nerve injury in rats. J Comp Neurol 2001;439:259-74.

57. Woodham P, Anderson PN, Nadim W, Turmaine M. Satellite cells surrounding axotomised rat dorsal root ganglion cells increase expression of a GFAP-like protein. Neurosci Lett 1989;98:8-12.

58. Nascimento RS, Santiago MF, Marques SA, Allodi S, Martinez AM. Diversity among satellite glial cells in dorsal root ganglia of the rat. Braz J Med Biol Res 2008;41:1011-7.

59. Sandelin M, Zabihi S, Liu L, Wicher G, Kozlova EN. Metastasisassociated S100A4 (Mts1) protein is expressed in subpopulations of sensory and autonomic neurons and in Schwann cells of the adult rat. J Comp Neurol 2004;473:233-43.

60. Vit JP, Ohara PT, Bhargava A, Kelley K, Jasmin L. Silencing the Kir4.1 potassium channel subunit in satellite glial cells of the rat trigeminal ganglion results in pain-like behavior in the absence of nerve injury. J Neurosci 2008;28:4161-71.

61. Keast JR, Stephensen TM. Glutamate and aspartate immunoreactivity in dorsal root ganglion cells supplying visceral and somatic targets and evidence for peripheral axonal transport. J Comp Neurol 2000;424:577-87.

62. Devor M, Wall PD. Cross-excitation in dorsal root ganglia of nerve-injured and intact rats. J Neurophysiol 1990;64:1733-46. 
63. Rozanski GM, Kim H, Li Q, Wong FK, Stanley EF. Slow chemical transmission between dorsal root ganglion neuron somata. Eur J Neurosci 2012;36:3314-21.

64. Rozanski GM, Li Q, Stanley EF. Transglial transmission at the dorsal root ganglion sandwich synapse: glial cell to postsynaptic neuron communication. Eur J Neurosci 2013;37:1221-8.

65. Belzer V, Hanani M. Nitric oxide as a messenger between neurons and satellite glial cells in dorsal root ganglia. Glia 2019;67:1296-307.

66. Hanz S, Fainzilber M. Retrograde signaling in injured nerve-the axon reaction revisited. J Neurochem 2006;99:13-9.

67. Scheib J, Hoke A. Advances in peripheral nerve regeneration. Nat Rev Neurol 2013;9:668-76.

68. Jortner BS. Common structural lesions of the peripheral nervous system. Toxicol Pathol 2020;48:96-104.

69. Carozzi VA, Canta A, Oggioni N, Sala B, Chiorazzi A, Meregalli C, et al. Neurophysiological and neuropathological characterization of new murine models of chemotherapyinduced chronic peripheral neuropathies. Exp Neurol 2010;226:301-9.

70. Figliuzzi M, Bianchi R, Cavagnini C, Lombardi R, PorrettaSerapiglia C, Lauria G, et al. Islet transplantation and insulin administration relieve long-term complications and rescue the residual endogenous pancreatic beta cells. Am J Pathol 2013; 183:1527-38.

71. Goncalves NP, Vaegter CB, Andersen H, Ostergaard L, Calcutt NA, Jensen TS. Schwann cell interactions with axons and microvessels in diabetic neuropathy. Nat Rev Neurol 2017;13:135-47.

72. Roman-Pintos LM, Villegas-Rivera G, Rodriguez-Carrizalez AD, Miranda-Diaz AG, Cardona-Munoz EG. Diabetic polyneuropathy in type 2 diabetes mellitus: Inflammation, oxidative stress, and mitochondrial function. J Diabetes Res 2016;2016:3425617.

73. Russell JW, Golovoy D, Vincent AM, Mahendru P, Olzmann JA, Mentzer A, et al. High glucose-induced oxidative stress and mitochondrial dysfunction in neurons. FASEB J 2002;16:1738-48.

74. Schmeichel AM, Schmelzer JD, Low PA. Oxidative injury and apoptosis of dorsal root ganglion neurons in chronic experimental diabetic neuropathy. Diabetes 2003;52:165-71.

75. Othman A, Bianchi R, Alecu I, Wei Y, Porretta-Serapiglia C, Lombardi R, et al. Lowering plasma 1-deoxysphingolipids improves neuropathy in diabetic rats. Diabetes 2015;64: 1035-45.

76. Zhang Q, Song W, Zhao B, Xie J, Sun Q, Shi X, et al. Quercetin attenuates diabetic peripheral neuropathy by correcting mitochondrial abnormality via activation of AMPK/PGC-1alpha pathway in vivo and in vitro. Front Neurosci 2021;15:636172.

77. Frias B, Merighi A. Capsaicin, nociception and pain. Molecules 2016;21:797.

78. Moran MM, McAlexander MA, Biro T, Szallasi A. Transient receptor potential channels as therapeutic targets. Nat Rev Drug Discov 2011;10:601-20.

79. Szallasi A, Cortright DN, Blum CA, Eid SR. The vanilloid receptor TRPV1: 10 years from channel cloning to antagonist proof-of-concept. Nat Rev Drug Discov 2007;6:357-72.

80. Szallasi A, Blumberg PM. Vanilloid (Capsaicin) receptors and mechanisms. Pharmacol Rev 1999;51:159-212.

81. Caterina MJ, Schumacher MA, Tominaga M, Rosen TA, Levine JD, Julius D. The capsaicin receptor: a heat-activated ion channel in the pain pathway. Nature 1997;389:816-24.

82. Quartu M, Carozzi VA, Dorsey SG, Serra MP, Poddighe L,
Picci $\mathrm{C}$, et al. Bortezomib treatment produces nocifensive behavior and changes in the expression of TRPV1, CGRP, and substance $P$ in the rat DRG, spinal cord, and sciatic nerve. Biomed Res Int 2014;2014:180428.

83. Fenzi F, Benedetti MD, Moretto G, Rizzuto N. Glial cell and macrophage reactions in rat spinal ganglion after peripheral nerve lesions: an immunocytochemical and morphometric study. Arch Ital Biol 2001;139:357-65.

84. Muratori L, Ronchi G, Raimondo S, Geuna S, GiacobiniRobecchi MG, Fornaro M. Generation of new neurons in dorsal root Ganglia in adult rats after peripheral nerve crush injury. Neural Plast 2015;2015:860546.

85. Pannese E. Number and structure of perisomatic satellite cells of spinal ganglia under normal conditions or during axon regeneration and neuronal hypertrophy. Z Zellforsch Mikrosk Anat 1964;63:568-92.

86. Takeda M, Tanimoto T, Kadoi J, Nasu M, Takahashi M, Kitagawa J, et al. Enhanced excitability of nociceptive trigeminal ganglion neurons by satellite glial cytokine following peripheral inflammation. Pain 2007;129:155-66.

87. Xian CJ, Zhou XF. Neuronal-glial differential expression of TGF-alpha and its receptor in the dorsal root ganglia in response to sciatic nerve lesion. Exp Neurol 1999;157:317-26.

88. Zhang Y, Roslan R, Lang D, Schachner M, Lieberman AR, Anderson PN. Expression of CHL1 and L1 by neurons and glia following sciatic nerve and dorsal root injury. Mol Cell Neurosci 2000;16:71-86.

89. Zhou XF, Rush RA, McLachlan EM. Differential expression of the p75 nerve growth factor receptor in glia and neurons of the rat dorsal root ganglia after peripheral nerve transection. J Neurosci 1996;16:2901-11.

90. Pannese E, Ledda M, Cherkas PS, Huang TY, Hanani M. Satellite cell reactions to axon injury of sensory ganglion neurons: increase in number of gap junctions and formation of bridges connecting previously separate perineuronal sheaths. Anat Embryol (Berl) 2003;206:337-47.

91. Hanani M, Huang TY, Cherkas PS, Ledda M, Pannese E. Glial cell plasticity in sensory ganglia induced by nerve damage. Neuroscience 2002;114:279-83.

92. Di Cesare Mannelli L, Pacini A, Micheli L, Tani A, Zanardelli M, Ghelardini C. Glial role in oxaliplatin-induced neuropathic pain. Exp Neurol 2014;261:22-33.

93. Warwick RA, Hanani M. The contribution of satellite glial cells to chemotherapy-induced neuropathic pain. Eur J Pain 2013; $17: 571-80$

94. Hanani M. Intercellular communication in sensory ganglia by purinergic receptors and gap junctions: implications for chronic pain. Brain Res 2012;1487:183-91.

95. Carozzi VA, Renn CL, Bardini M, Fazio G, Chiorazzi A, Meregalli C, et al. Bortezomib-induced painful peripheral neuropathy: an electrophysiological, behavioral, morphological and mechanistic study in the mouse. PLoS One 2013;8:e72995.

96. Cece R, Petruccioli MG, Pizzini G, Cavaletti G, Tredici G. Ultrastructural aspects of DRG satellite cell involvement in experimental cisplatin neuronopathy. J Submicrosc Cytol Pathol 1995;27:417-25.

97. Abdelhak A, Huss A, Kassubek J, Tumani H, Otto M. Serum GFAP as a biomarker for disease severity in multiple sclerosis. Sci Rep 2018;8:14798.

98. Hanani M, Blum E, Liu S, Peng L, Liang S. Satellite glial cells in dorsal root ganglia are activated in streptozotocintreated rodents. J Cell Mol Med 2014;18:2367-71.

99. Nascimento DS, Castro-Lopes JM, Moreira Neto FL. Satellite glial cells surrounding primary afferent neurons are 
activated and proliferate during monoarthritis in rats: is there a role for ATF3? PLoS One 2014;9:e108152.

100. Schindler CR, Lustenberger T, Woschek M, Stormann P, Henrich D, Radermacher $\mathrm{P}$, et al. Severe traumatic brain injury (TBI) modulates the kinetic profile of the inflammatory response of markers for neuronal damage. J Clin Med 2020;9:1667.

101. Yuan Q, Liu X, Xian YF, Yao M, Zhang X, Huang P, et al. Satellite glia activation in dorsal root ganglion contributes to mechanical allodynia after selective motor fiber injury in adult rats. Biomed Pharmacother 2020;127:110187.

102. Adaes S, Almeida L, Potes CS, Ferreira AR, Castro-Lopes JM, Ferreira-Gomes J, et al. Glial activation in the collagenase model of nociception associated with osteoarthritis. Mol Pain 2017;13:1744806916688219.

103. Barragan-Iglesias P, Oidor-Chan VH, Loeza-Alcocer E, Pineda-Farias JB, Velazquez-Lagunas I, Salinas-Abarca AB, et al. Evaluation of the neonatal streptozotocin model of diabetes in rats: Evidence for a model of neuropathic pain. Pharmacol Rep 2018;70:294-303.

104. Peters CM, Jimenez-Andrade JM, Kuskowski MA, Ghilardi JR, Mantyh PW. An evolving cellular pathology occurs in dorsal root ganglia, peripheral nerve and spinal cord following intravenous administration of paclitaxel in the rat. Brain Res 2007;1168:46-59.

105. Kalynovska N, Diallo M, Sotakova-Kasparova D, Palecek J. Losartan attenuates neuroinflammation and neuropathic pain in paclitaxel-induced peripheral neuropathy. J Cell Mol Med 2020;24:7949-58.

106. Zhang $\mathrm{H}$, Li Y, de Carvalho-Barbosa M, Kavelaars A, Heijnen CJ, Albrecht PJ, et al. Dorsal root ganglion infiltration by macrophages contributes to paclitaxel chemotherapyinduced peripheral neuropathy. J Pain 2016;17:775-86.

107. Hu P, McLachlan EM. Macrophage and lymphocyte invasion of dorsal root ganglia after peripheral nerve lesions in the rat. Neuroscience 2002;112:23-38.

108. Xie WR, Deng H, Li H, Bowen TL, Strong JA, Zhang JM. Robust increase of cutaneous sensitivity, cytokine production and sympathetic sprouting in rats with localized inflammatory irritation of the spinal ganglia. Neuroscience 2006;142:809-22.

109. Kamiya H, Zhang W, Sima AA. Degeneration of the Golgi and neuronal loss in dorsal root ganglia in diabetic BioBreeding/Worcester rats. Diabetologia 2006;49:2763-74.

110. Russell JW, Sullivan KA, Windebank AJ, Herrmann DN, Feldman EL. Neurons undergo apoptosis in animal and cell culture models of diabetes. Neurobiol Dis 1999;6:347-63.

111. Scuteri A, Galimberti A, Maggioni D, Ravasi M, Pasini S, Nicolini G, et al. Role of MAPKs in platinum-induced neuronal apoptosis. Neurotoxicology 2009;30:312-9.

112. Bobylev I, Joshi AR, Barham M, Neiss WF, Lehmann HC. Depletion of mitofusin-2 causes mitochondrial damage in cisplatin-induced neuropathy. Mol Neurobiol 2018;55:1227-35.

113. Canta A, Pozzi E, Carozzi VA. Mitochondrial dysfunction in chemotherapy-induced peripheral neuropathy (CIPN). Toxics 2015;3:198-223.

114. Leo M, Schmitt LI, Kusterarent P, Kutritz A, Rassaf T, Kleinschnitz C, et al. Platinum-based drugs cause mitochondrial dysfunction in cultured dorsal root ganglion neurons. Int J Mol Sci 2020;21:8636.

115. McDonald ES, Randon KR, Knight A, Windebank AJ. Cisplatin preferentially binds to DNA in dorsal root ganglion neurons in vitro and in vivo: a potential mechanism for neurotoxicity. Neurobiol Dis 2005;18:305-13.

116. Ta LE, Espeset L, Podratz J, Windebank AJ. Neurotoxicity of oxaliplatin and cisplatin for dorsal root ganglion neurons correlates with platinum-DNA binding. Neurotoxicology 2006;27:992-1002.

117. Podratz JL, Knight AM, Ta LE, Staff NP, Gass JM, Genelin $\mathrm{K}$, et al. Cisplatin induced mitochondrial DNA damage in dorsal root ganglion neurons. Neurobiol Dis 2011;41:661-8.

118. Corsetti G, Rodella L, Rezzani R, Stacchiotti A, Bianchi R. Cytoplasmic changes in satellite cells of spinal ganglia induced by cisplatin treatment in rats. Ultrastruct Pathol 2000;24:259-65.

119. Garrett FG, Durham PL. Differential expression of connexins in trigeminal ganglion neurons and satellite glial cells in response to chronic or acute joint inflammation. Neuron Glia Biol 2008;4:295-306.

120. Ohara PT, Vit JP, Bhargava A, Jasmin L. Evidence for a role of connexin 43 in trigeminal pain using RNA interference in vivo. J Neurophysiol 2008;100:3064-73.

121. Verkhratsky A, Fernyhough P. Calcium signalling in sensory neurones and peripheral glia in the context of diabetic neuropathies. Cell Calcium 2014;56:362-71.

122. Liu S, Zou L, Xie J, Xie W, Wen S, Xie Q, et al. LncRNA NONRATT021972 siRNA regulates neuropathic pain behaviors in type 2 diabetic rats through the $\mathrm{P} 2 \mathrm{X} 7$ receptor in dorsal root ganglia. Mol Brain 2016;9:44.

123. Porter DDL, Morton PD. Clearing techniques for visualizing the nervous system in development, injury, and disease. J Neurosci Methods 2020;334:108594.

124. Ueda HR, Dodt HU, Osten P, Economo MN, Chandrashekar J, Keller PJ. Whole-brain profiling of cells and circuits in mammals by tissue clearing and light-sheet microscopy. Neuron 2020;106:369-87.

125. Ahnfelt-Ronne J, Jorgensen MC, Hald J, Madsen OD, Serup P, Hecksher-Sorensen J. An improved method for threedimensional reconstruction of protein expression patterns in intact mouse and chicken embryos and organs. J Histochem Cytochem 2007;55:925-30.

126. Renier N, Wu Z, Simon DJ, Yang J, Ariel P, Tessier-Lavigne M. iDISCO: a simple, rapid method to immunolabel large tissue samples for volume imaging. Cell 2014;159:896-910.

127. West SJ, Bennett DL. StereoMate: A stereological and automated analysis platform for assessing histological labelling in cleared tissue specimens. bioRxiv 2019:648337.

128. Chung K, Deisseroth K. CLARITY for mapping the nervous system. Nat Methods 2013;10:508-13.

129. Yang B, Treweek JB, Kulkarni RP, Deverman BE, Chen CK, Lubeck E, et al. Single-cell phenotyping within transparent intact tissue through whole-body clearing. Cell 2014;158:945-58.

130. Bernal L, Cisneros E, Garcia-Magro N, Roza C. Immunostaining in whole-mount lipid-cleared peripheral nerves and dorsal root ganglia after neuropathy in mice. Sci Rep 2019;9:8374.

131. Ciglieri E, Ferrini F, Boggio E, Salio C. An improved method for in vitro morphofunctional analysis of mouse dorsal root ganglia. Ann Anat 2016;207:62-7.

132. Hayar A, Gu C, Al-Chaer ED. An improved method for patch clamp recording and calcium imaging of neurons in the intact dorsal root ganglion in rats. $\mathrm{J}$ Neurosci Methods 2008; 173:74-82.

133. Zhang JM, Donnelly DF, LaMotte RH. Patch clamp recording from the intact dorsal root ganglion. J Neurosci Methods 1998;79:97-103.

134. Tomer R, Ye L, Hsueh B, Deisseroth K. Advanced CLARITY for rapid and high-resolution imaging of intact tissues. Nat Protoc 2014;9:1682-97. 
135. Quinta HR, Pasquini LA, Pasquini JM. Three-dimensional reconstruction of corticospinal tract using one-photon confocal microscopy acquisition allows detection of axonal disruption in spinal cord injury. J Neurochem 2015;133:113-24.

136. Di Cataldo S, Tonti S, Ciglieri E, Ferrini F, Macii E, Ficarra E, et al. Automated 3D immunofluorescence analysis of dorsal root ganglia for the investigation of neural circuit alterations: a preliminary study. Ann Comp Sci Inform Syst 2016;9:65-70.
137. Piccinini F, Balassa T, Carbonaro A, Diosdi A, Toth T, Moshkov N, et al. Software tools for 3D nuclei segmentation and quantitative analysis in multicellular aggregates. Comput Struct Biotechnol J 2020;18:1287-300.

138. Ferrini F, Russo A, Salio C. Fos and pERK immunoreactivity in spinal cord slices: Comparative analysis of in vitro models for testing putative antinociceptive molecules. Ann Anat 2014;196:217-23.

Received for publication: 26 May 2021. Accepted for publication: 16 August 2021.

This work is licensed under a Creative Commons Attribution-NonCommercial 4.0 International License (CC BY-NC 4.0).

(C) Copyright: the Author(s), 2021

Licensee PAGEPress, Italy

European Journal of Histochemistry 2021; 65(s1):3276

doi:10.4081/ejh.2021.3276 